\title{
Thoracolumbar kyphoscoliosis with unilateral subluxation of the spine and postoperative lumbar spondylolisthesis in Hunter syndrome
}

\author{
Simon B. Roberts, MRCS, and Athanasios I. Tsirikos, FRCS \\ Scottish National Spine Deformity Centre, Royal Hospital for Sick Children, Edinburgh, United Kingdom
}

Surgical correction for kyphoscoliosis is increasingly being performed for patients with mucopolysaccharidosis (MPS). Reported case series have predominantly included patients with Type I (Hurler) and Type IV (Morquio) MPS. To their knowledge, the authors describe the first case report of surgical management of thoracolumbar kyphoscoliosis in Hunter syndrome (MPS Type II) and the rare occurrence of lumbar spondylolisthesis following surgical stabilization. A 12-yearold boy with Hunter syndrome presented with severe thoracolumbar kyphoscoliosis and no associated symptoms. Spinal radiographs demonstrated kyphosis of $48^{\circ}(\mathrm{T} 11-\mathrm{L} 3)$ and scoliosis of $22^{\circ}(\mathrm{T} 11-\mathrm{L} 3)$ with an anteriorly hypoplastic L-1 vertebra. The deformity progressed to kyphosis of $60^{\circ}$ and scoliosis of $42^{\circ}$ prior to surgical intervention. Spinal CT scans identified left T12-L1 facet subluxation, causing anterior rotatory displacement of the spine proximal to L-1 and bilateral L-5 isthmic spondylolysis with no spondylolisthesis. A combined single-stage anterior and posterior instrumented spinal arthrodesis from T-9 to L-4 was performed. Kyphosis and scoliosis were corrected to $4^{\circ}$ and $0^{\circ}$, respectively. Prolonged ventilator support and nasogastric feedings were required for 3 months postoperatively. At 2.5 years following surgery, the patient was asymptomatic, mobilizing independently, and had achieved a solid spinal fusion. However, he had also developed a Grade II spondylolisthesis at L4-5; this was managed nonoperatively in the absence of symptoms or further deterioration of the spondylolisthesis to the 3.5-year postoperative follow-up visit. Satisfactory correction of thoracolumbar kyphoscoliosis in Hunter syndrome can be achieved by combined anterior/posterior instrumented arthrodesis. The risk of developing deformity or instability in motion segments adjacent to an instrumented fusion may be greater in patients with MPS related to the underlying connective tissue disorder.

http://thejns.org/doi/abs/10.3171/2015.6.SPINE15268

KEY WORDS thoracolumbar; kyphoscoliosis; Hunter syndrome; spondylolisthesis; mucopolysaccharidosis; deformity

S URGICAL correction for kyphoscoliosis is increasingly being performed in patients with mucopolysaccharidosis (MPS), whose life expectancy has improved since the advent of enzyme replacement therapy and hematopoietic stem cell transplantation..$^{10}$ The musculoskeletal manifestations of MPS, including Hunter syndrome, are largely unresponsive to these treatments. ${ }^{3,8,11}$ Reported case series of MPS patients have predominantly included individuals with Type I (Hurler) and Type IV (Morquio) MPS and have described high incidences of thoracolumbar kyphosis and associated L1-2 spondylolisthesis at the deformity apex. ${ }^{1,5,13,16} \mathrm{We}$ describe the first report of surgical correction of thoracolumbar kyphosis in a patient with MPS Type II (Hunter syndrome) and the management of a rare complication, postoperative adjacent-segment spondylolisthesis.

\section{Case Report}

History and Examination

A 12-year-old boy with Hunter syndrome presented with a severe thoracolumbar kyphoscoliosis of 1-year duration and no associated symptoms. The patient's previous medical history included iduronate-2-sulfatase therapy starting when he was 5 years old, mild mitral valve regurgitation, tracheolaryngomalacia, persistent toe-walking with flexed posture, bilateral equinovarus and cavus feet treated by soft-tissue release, wheelchair use for prolonged mobilization, and severe behavioral problems that limited compliance with medical treatment (Table 1).

Clinical examination demonstrated a rigid acute thoracolumbar kyphosis with associated mild thoracolumbar scoliosis. Neurological examination demonstrated normal 
TABLE 1. Clinical manifestations in our patient

\begin{tabular}{ll}
\hline \multicolumn{1}{c}{ Musculoskeletal } & \multicolumn{1}{c}{ Nonmusculoskeletal } \\
\hline Periodontoid soft-tissue hyperpla- & Progressive cognitive impairment \\
sia (no cord compression) & Sensorineural hearing loss \\
Bilateral hip dysplasia & Mild mitral valve regurgitation \\
Bilateral equinovarus \& pes cavus & Tracheolaryngomalacia \\
\hline
\end{tabular}

muscle tone, muscle power, sensation, and deep tendon reflexes in the upper and lower limbs, within the limitations of the underlying behavioral disorder. There were no signs of spinal dysraphism, and the patient had no bowel or bladder dysfunction. Spinal radiographs (obtained in the erect sitting position due to poor patient compliance) demonstrated kyphosis of $48^{\circ}$ (from T-11 to L-3) and scoliosis of $22^{\circ}$ (from T-11 to L-3) with an anteriorly hypoplastic L-1 vertebra. Surgical stabilization of thoracolumbar kyphosis was scheduled and was performed 6 months after presentation, during which medical comorbidities were optimized and an elective preoperative tracheotomy was established. The thoracolumbar deformity progressed to $60^{\circ}$ of kyphosis and $42^{\circ}$ of scoliosis prior to surgical stabilization (Fig. 1). Spinal CT scans revealed subluxation of the left T12-L1 joint causing segmental instability, rotatory displacement of the spine proximal to L-1, and bilateral L-5 isthmic spondylolysis with no associated lumbosacral spondylolisthesis (Fig. 2). Spinal MRI revealed disc herniations at the T12-L1 and L1-2 levels that contacted both the conus medullaris and cauda equina (Fig. 3). MRI also demonstrated mild canal stenosis at the craniocervical junction due to periodontoid soft-tissue hyperplasia but no cord compression. During intubation, hyperextension of the cervical spine was avoided.
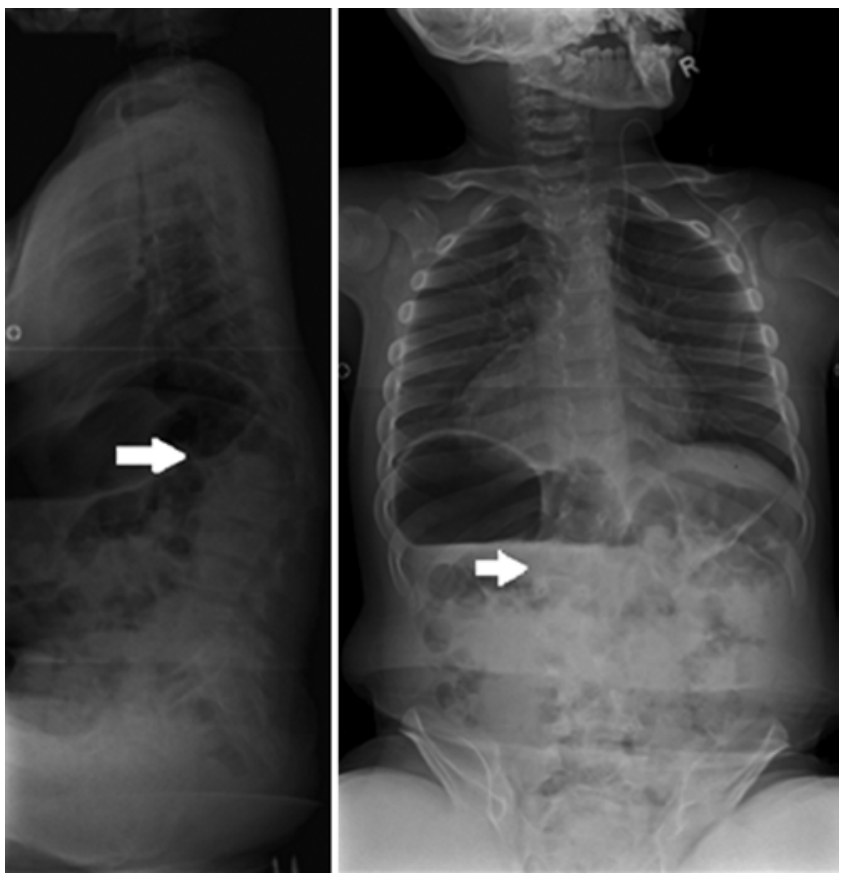

FIG. 1. Preoperative radiographs demonstrating T11-L3 kyphosis of $60^{\circ}$ with an anomalous L-1 vertebra (arrow, left) and T11-L3 scoliosis of $42^{\circ}$ convex to left (arrow, right).
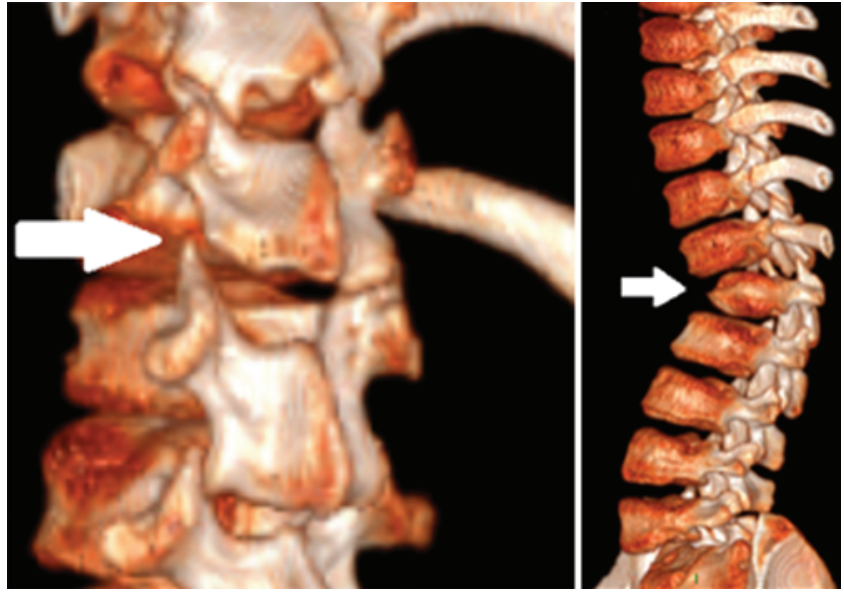

FIG. 2. Three-dimensional reconstructed CT images showing subluxation of the left T12-L1 facet joint (left) and displacement of the spine proximal to the hypoplastic L-1 vertebra (arrows) (right). Figure is available in color online only.

\section{Operation}

A combined single-stage anterior and posterior instrumented spinal arthrodesis from T-9 to L-4 was performed. Instrumented fusion extended from 2 levels cranial to 1 level caudal to the levels of the thoracolumbar deformity to achieve a stable correction of deformity, preserve distal motion segments, and avoid fixation to the sacrum/pelvis, thereby also preserving the patient's capacity to ambulate. An anterior thoracoabdominal approach permitted complete discectomies and single-rod instrumentation with transvertebral screws across T-10 to L-3. The cartilaginous, hypoplastic anterior L-1 vertebral body was excised and bridging rib struts were placed across T-12 to L-2 to allow apical correction of kyphosis and a solid fusion. A posterior segmental pedicle screw instrumented arthrodesis was then performed extending from T-9 to L-4 with autologous rib and allograft bone placed between the facets; instrumentation used comprised Universal Spine System II anterior instrumentation with anterior segmental transvertebral screws and a single $6.0-\mathrm{mm}$ diameter titanium rod (DePuy Synthes), and Universal Spine System II posterior instrumentation with segmental $5.0-\mathrm{mm}$ diameter pedicle screws and two 6.0-mm diameter titanium rods (DePuy Synthes). Kyphosis and scoliosis were corrected to $4^{\circ}$ and $0^{\circ}$, respectively (Fig. 4). Intraoperative multimodal spinal cord monitoring was performed to record transcranial motor evoked potentials for the upper and lower limbs and cortical/cervical somatosensory evoked potentials, which remained stable throughout the surgical procedures.

\section{Postoperative Course}

Prolonged ventilatory support (invasive and noninvasive) and nasogastric feedings were required for 3 months postoperatively. The tracheotomy was closed 3 months after surgery. At 2.5 years of follow-up, the patient was asymptomatic, mobilizing independently but with a markedly propulsive gait with both hip and knee flexion (also present preoperatively), and had achieved a solid circumferential spinal fusion, but had also developed a Grade II 


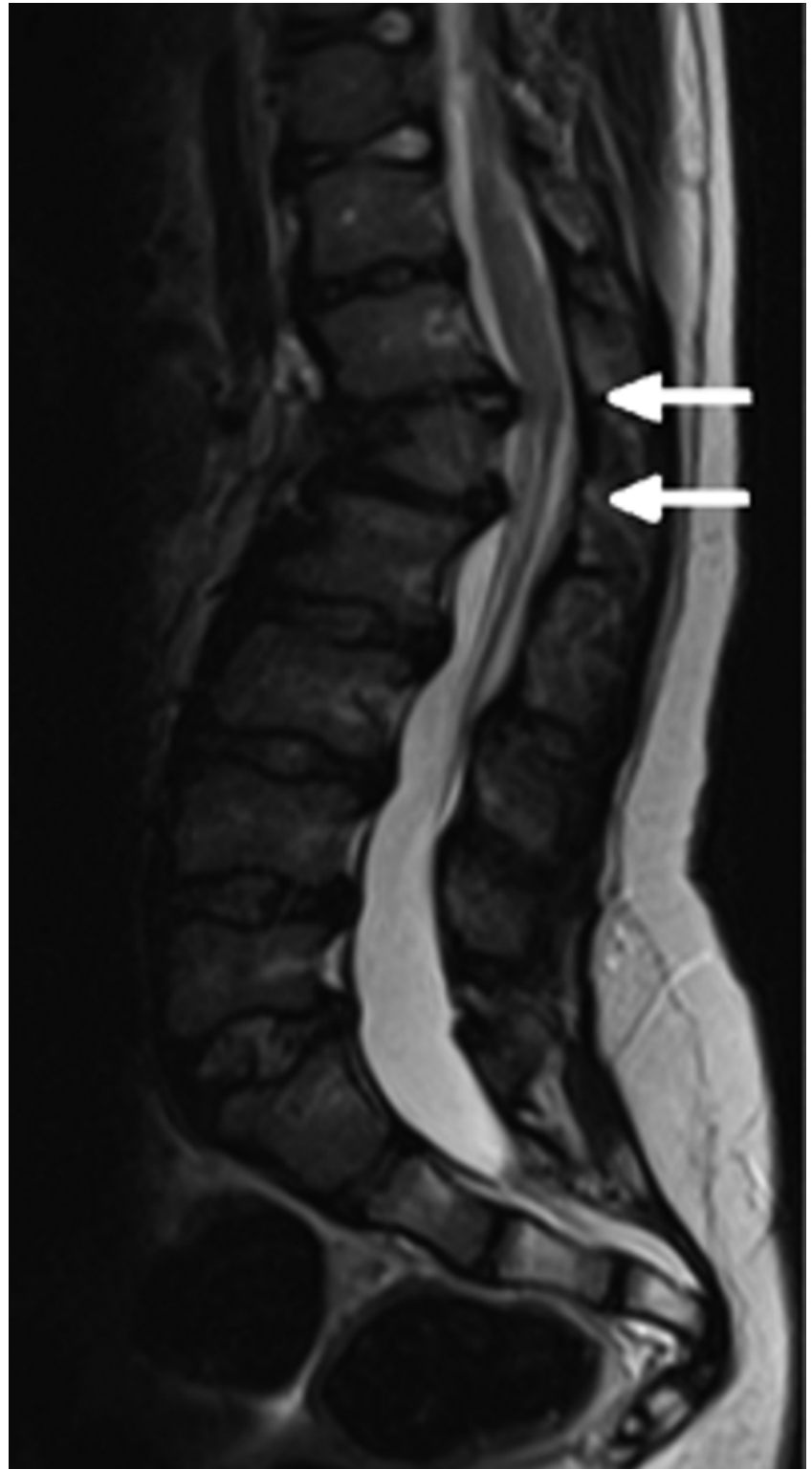

FIG. 3. Midsagittal MR image of the spine showing T12-L1 and L1-2 disc herniations (arrows) contacting the conus medullaris and cauda equina but causing no compression.

spondylolisthesis at the adjacent distal L4-5 level (Fig. 5). In the absence of symptoms or any further progression of spondylolisthesis to 3.5 years of follow-up, a decision was made by the parents against distal extension of the fusion and to continue conservative management. The patient has since developed carpal tunnel syndrome, which remains under review awaiting treatment.

\section{Discussion}

To our knowledge, this case is the first report of a patient with kyphoscoliosis associated with Hunter syndrome. Indications for surgery included the presence of a progressive severe thoracolumbar deformity with apical segmental instability and a high risk of subsequent neurological
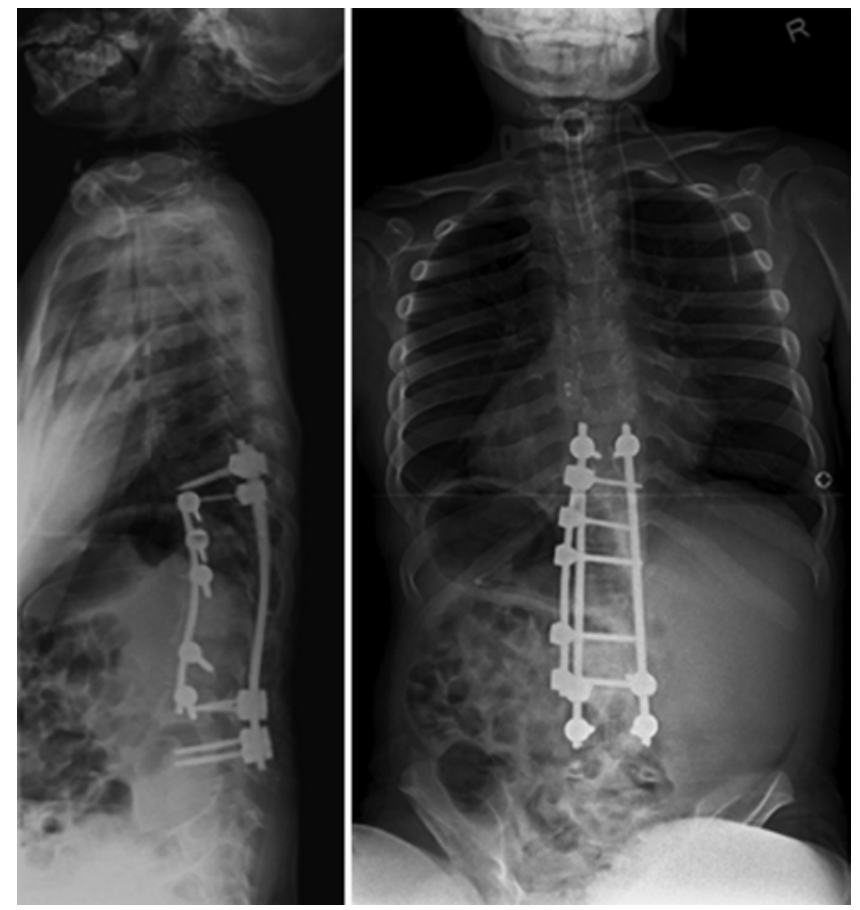

FIG. 4. Whole spine radiographs obtained immediately postoperatively, demonstrating excellent correction of the deformity to $4^{\circ}$ of kyphosis (left) and $0^{\circ}$ of scoliosis (right).

deterioration. This case report demonstrates that, in the management of severe thoracolumbar kyphosis associated with Hunter syndrome, satisfactory deformity correction and patient outcomes can be achieved by combined anterior/posterior instrumented arthrodesis, as has been demonstrated previously for other MPS subtypes. ${ }^{1,5,13,16}$

Spondylolisthesis is a recognized but infrequent occurrence following fusion for lumbar disc disease, adolescent idiopathic scoliosis, and congenital scoliosis., ${ }^{2,6,7,9,12,14}$ To our knowledge, this is the first report of spondylolisthesis developing after instrumented fusion for syndromic kyphoscoliosis, although this may not be unexpected in a patient with MPS. Coexisting lumbar spondylolysis/ spondylolisthesis and kyphosis or scoliosis are generally considered and managed separately. ${ }^{4}$ In this patient, fusion was performed to address the thoracolumbar kyphoscoliosis and segmental instability. Contributing factors in the development of spondylolisthesis following surgery in this case likely include the patient's propulsive gait and flexed posture, reduced connective tissue strength associated with MPS, and load concentration in the remaining lumbar motion segments following arthrodesis. ${ }^{15}$ Interestingly, the spondylolisthesis developed at the adjacent distal L4-5 level and not at the level below, at which preoperative spinal CT demonstrated bilateral L-5 spondylolysis that would also predispose to segmental instability.

Options to manage the postoperative lumbar spondylolisthesis in this case included either conservative treatment or surgical stabilization. Surgical stabilization of the L4-5 spondylolisthesis would require fusion with extension of the previous instrumentation to the sacrum and pelvis, which could jeopardize the patient's ability to ambulate. A 


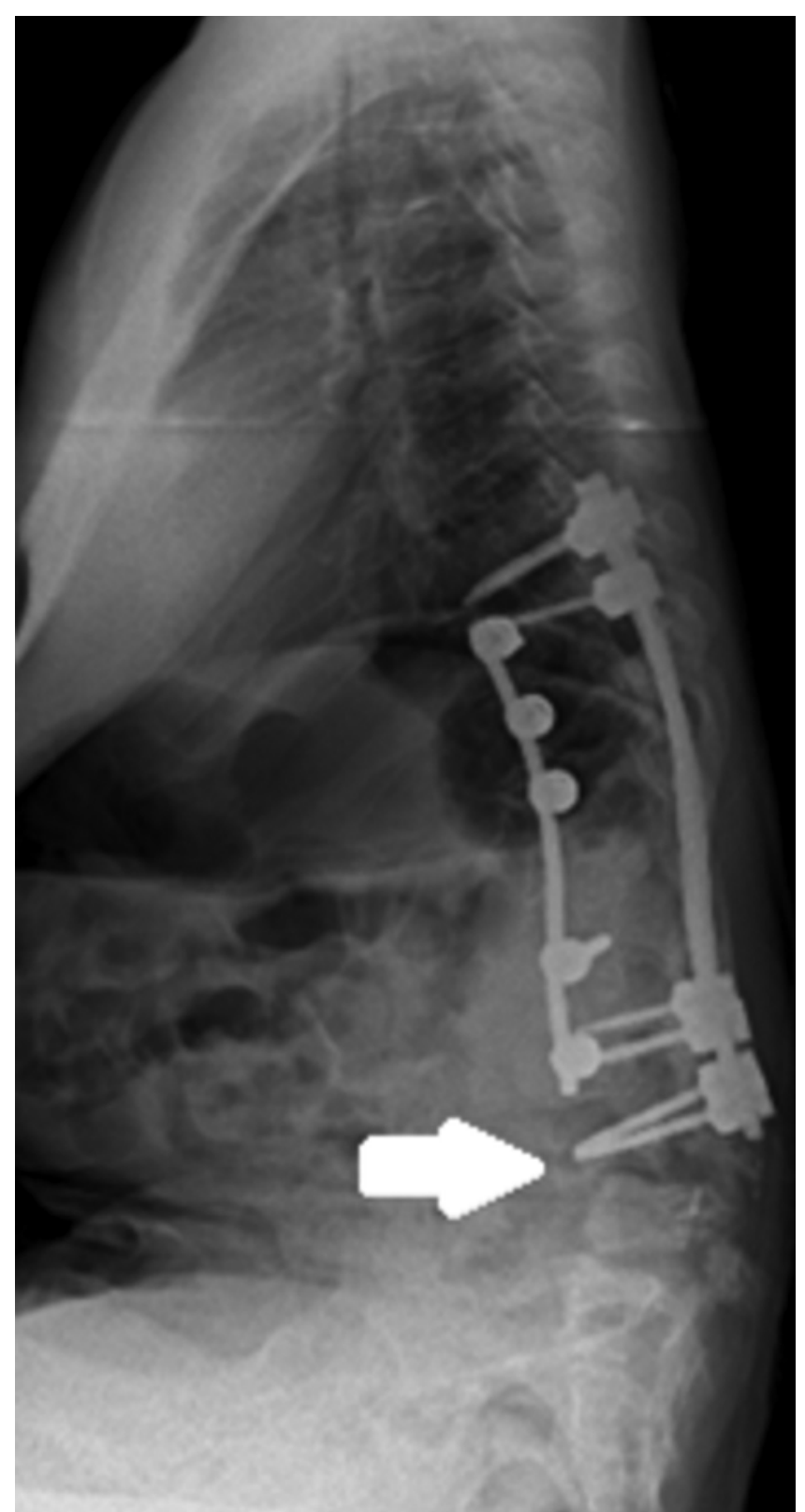

FIG. 5. Whole-spine lateral radiograph obtained 2.5 years postoperatively, when the patient was skeletally mature, showing no loss of kyphoscoliosis correction. Note the development of a Grade II L4-5 spondylolisthesis (arrow)

decision was made with the family to pursue conservative management given that the patient was asymptomatic and ambulating, and previous operations had been associated with significant risks, requiring elective tracheotomy and prolonged ventilator support postoperatively. The spondylolisthesis below the previously surgically treated levels remained stable to the end of skeletal growth. This case report highlights the risk of developing further deformity or segmental instability, producing spondylolisthesis below a previously performed instrumented spinal fusion in patients with connective tissue disorders, such as MPS. Patients with MPS may be at particularly high risk of such complications, ${ }^{16}$ especially as these patients are normally in severe flexed posture and marked positive sagittal imbalance. ${ }^{1}$ The extent of instrumented spinal arthrodesis to correct and stabilize a severe progressive thoracolumbar kyphosis in patients with Hunter syndrome should therefore include 1-2 spinal levels cranial to and caudal to the extent of deformity and avoid a distal fusion beyond the L-4 vertebra in patients with ambulatory capacity. In nonambulatory patients, consideration should be given to primary arthrodesis, extending distally to the sacrum/pelvis to preclude the development of distal segmental instability or progressive deformity postoperatively.

This case report demonstrates that rigid angular kyphoscoliosis associated with Hunter syndrome can be effectively managed by combined anterior/posterior instrumented arthrodesis and produce adequate correction and a stable fusion. Major medical comorbidities, airway anomalies, and respiratory compromise can significantly complicate and prolong the postoperative course. Spondylolisthesis distal to a previous instrumented fusion is a rare occurrence following deformity correction. This case highlights the possibility that the risk of developing deformity or instability in motion segments adjacent to an instrumented fusion may be greater in patients with connective tissue disorders such as MPS.

\section{References}

1. Abelin Genevois K, Garin C, Solla F, Guffon N, Kohler R: Surgical management of thoracolumbar kyphosis in mucopolysaccharidosis type 1 in a reference center. J Inherit Metab Dis 37:69-78, 2014

2. Anderson CE: Spondyloschisis following spine fusion. J Bone Joint Surg Am 38-A:1142-1146, 1956

3. Breider MA, Shull RM, Constantopoulos G: Long-term effects of bone marrow transplantation in dogs with mucopolysaccharidosis I. Am J Pathol 134:677-692, 1989

4. Crostelli M, Mazza O: AIS and spondylolisthesis. Eur Spine J 22 (Suppl 2):S172-S184, 2013

5. Dalvie SS, Noordeen MH, Vellodi A: Anterior instrumented fusion for thoracolumbar kyphosis in mucopolysaccharidosis. Spine (Phila Pa 1976) 26:E539-E541, 2001

6. Depalma AF, Marone PJ: Spondylolysis following spinal fusion. Report of a case. Clin Orthop 15:208-211, 1959

7. Friedman RJ, Micheli LJ: Acquired spondylolisthesis following scoliosis surgery. A case report. Clin Orthop Relat Res (190):132-134, 1984

8. Guffon N, Bertrand Y, Forest I, Fouilhoux A, Froissart R: Bone marrow transplantation in children with Hunter syndrome: outcome after 7 to 17 years. J Pediatr 154:733-737, 2009

9. Harrington PR, Tullos HS: Spondylolisthesis in children. Observations and surgical treatment. Clin Orthop Relat Res 79:75-84, 1971

10. Hobbs JR: Bone marrow transplantation for inborn errors. Lancet 2:735-739, 1981

11. Link B, de Camargo Pinto LL, Giugliani R, Wraith JE, Guffon N, Eich E, et al: Orthopedic manifestations in patients with mucopolysaccharidosis type II (Hunter syndrome) enrolled in the Hunter Outcome Survey. Orthop Rev (Pavia) 2:e16, 2010

12. Rombold C: Spondylolysis: a complication of spine fusion. $\mathbf{J}$ Bone Joint Surg Am 47:1237-1242, 1965

13. Tandon V, Williamson JB, Cowie RA, Wraith JE: Spinal problems in mucopolysaccharidosis I (Hurler syndrome). J Bone Joint Surg Br 78:938-944, 1996 
14. Tietjen R, Morgenstern JM: Spondylolisthesis following surgical fusion for scoliosis: a case report. Clin Orthop Relat Res (117):176-178, 1976

15. Tsirikos AI, Garrido EG: Spondylolysis and spondylolisthesis in children and adolescents. J Bone Joint Surg Br 92:751-759, 2010

16. Yasin MN, Sacho R, Oxborrow NJ, Wraith JE, Williamson JB, Siddique I: Thoracolumbar kyphosis in treated mucopolysaccharidosis 1 (Hurler syndrome). Spine (Phila Pa 1976) 39:381-387, 2014

\section{Disclosures}

The authors report no conflict of interest concerning the materials or methods used in this study or the findings specified in this paper.

\section{Author Contributions}

Conception and design: both authors. Acquisition of data: both authors. Analysis and interpretation of data: both authors. Drafting the article: both authors. Critically revising the article: both authors. Reviewed submitted version of manuscript: both authors. Approved the final version of the manuscript on behalf of both authors: Roberts.

\section{Supplemental Information}

Previous Presentations

Portions of this work were presented in poster form at the British Scoliosis Society annual meeting in Sheffield (United Kingdom) on April 22-23, 2015.

\section{Correspondence}

Simon B. Roberts, University of Edinburgh/Scottish National Spine Deformity Centre, Royal Hospital for Sick Children, Sciennes Rd., Edinburgh, Midlothian EH9 1LF, United Kingdom. email: simon.roberts@ed.ac.uk. 